\title{
Calculation of System International Sensitivity Index: how many calibrant plasmas are required?
}

\author{
J M K Dufty, S Craig, K J Stevenson, D A Taberner
}

Thrombosis Reference

Centre,

Withington Hospital,

Manchester M20 2LR

Correspondence to:

Dr D A Taberner.

Accepted for publication 22 October 1996

Table 1 Systems used

Table 2 Systems grouped as other

\begin{tabular}{lll}
\hline Coagulometer & Thromboplastin reagent & Number of laboratories \\
\hline Sysmex & Low opacity Manchester reagent & 3 \\
Sysmex & Diagen Activated & 2 \\
Sysmex & Manchester reagent & 1 \\
Sysmex & Dade IS & 1 \\
Sysmex & Dade Innovin & 1 \\
Coag-a-Mate & Manchester reagent & 2 \\
MLA Electra & Manchester reagent & 2 \\
Hemolab Biomerieux & Low opacity Manchester reagent & 1 \\
Total & & 13 \\
\hline
\end{tabular}
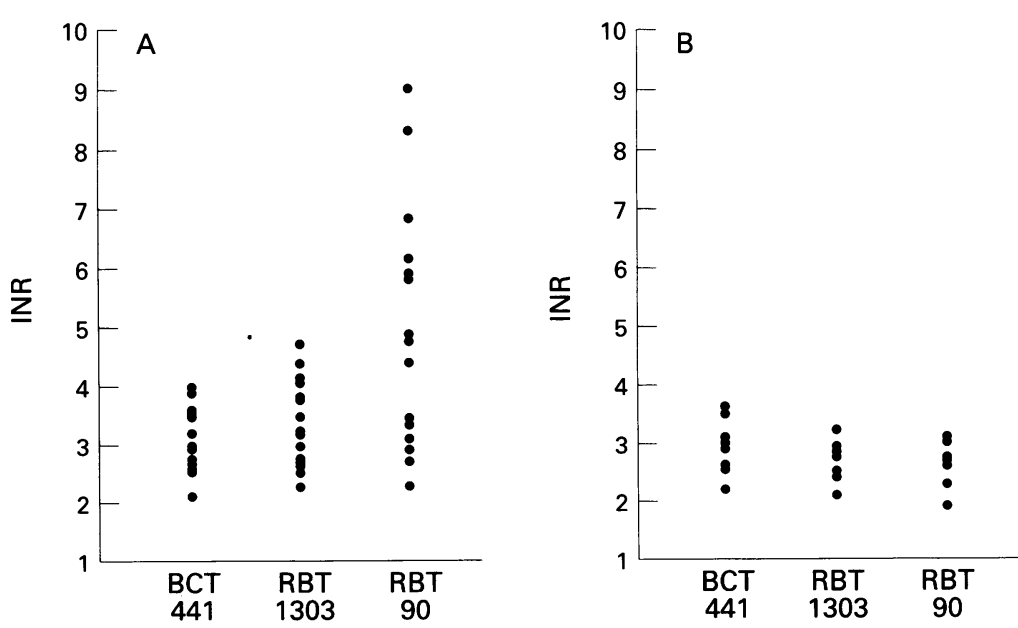

Figure 1 INR values of adsorbed and coumarin plasmas with three thromboplastin reagents.

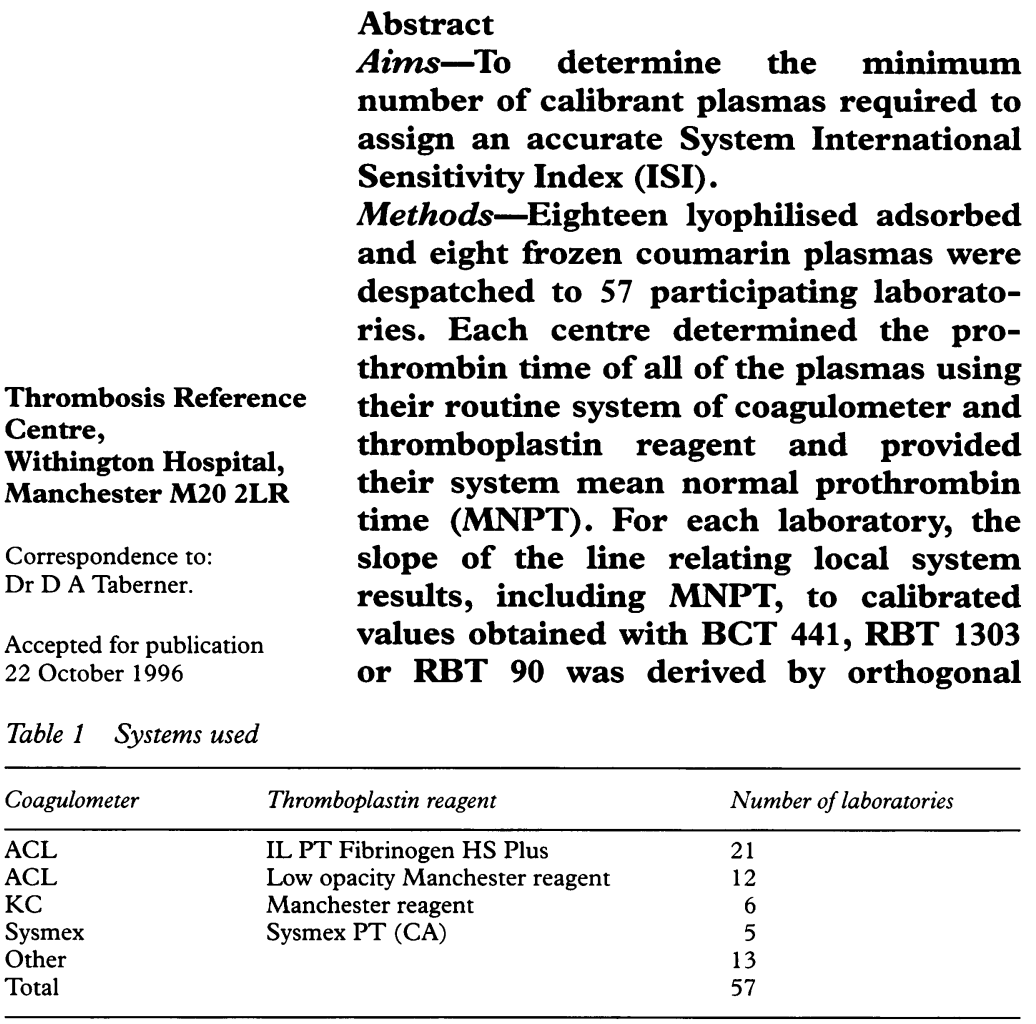

Coagulometer induced errors in the reporting of International Normalised Ratios (INR) may be controlled by assigning a System ISI-that is, a corrected ISI specific for the combination or System of coagulometer and thromboplastin reagent in local use.

One approach to the calculation of the System ISI is to determine the prothrombin time, with the local coagulometer/thromboplastin combination, of a set of calibrant plasmas and to relate these values to those obtained manually with reference thromboplastins, by orthogonal regression. ${ }^{2}$

To minimise the work involved in routine coagulation laboratories, an abbreviated version of the established World Health Organisation (WHO) calibrating procedure has been used, since 1991, in the Thrombosis Reference Centre System ISI Calibrant plasma kit. In this method a set of 18 plasmas covering the therapeutic range for oral anticoagulation is prepared from normal plasma by adsorption of the vitamin $\mathrm{K}$ dependent clotting factors onto barium sulphate. These adsorbed plasmas have a calibrated value assigned following prothrombin time testing by the manual method, using reference thromboplastins, including WHO International Reference Preparations (IRP) and BCR Certified Reference Materials. They are then used in the local coagulometer/ thromboplastin system. The data obtained from these 18 plasmas replaces those from 60 patient plasmas recommended by the WHO 

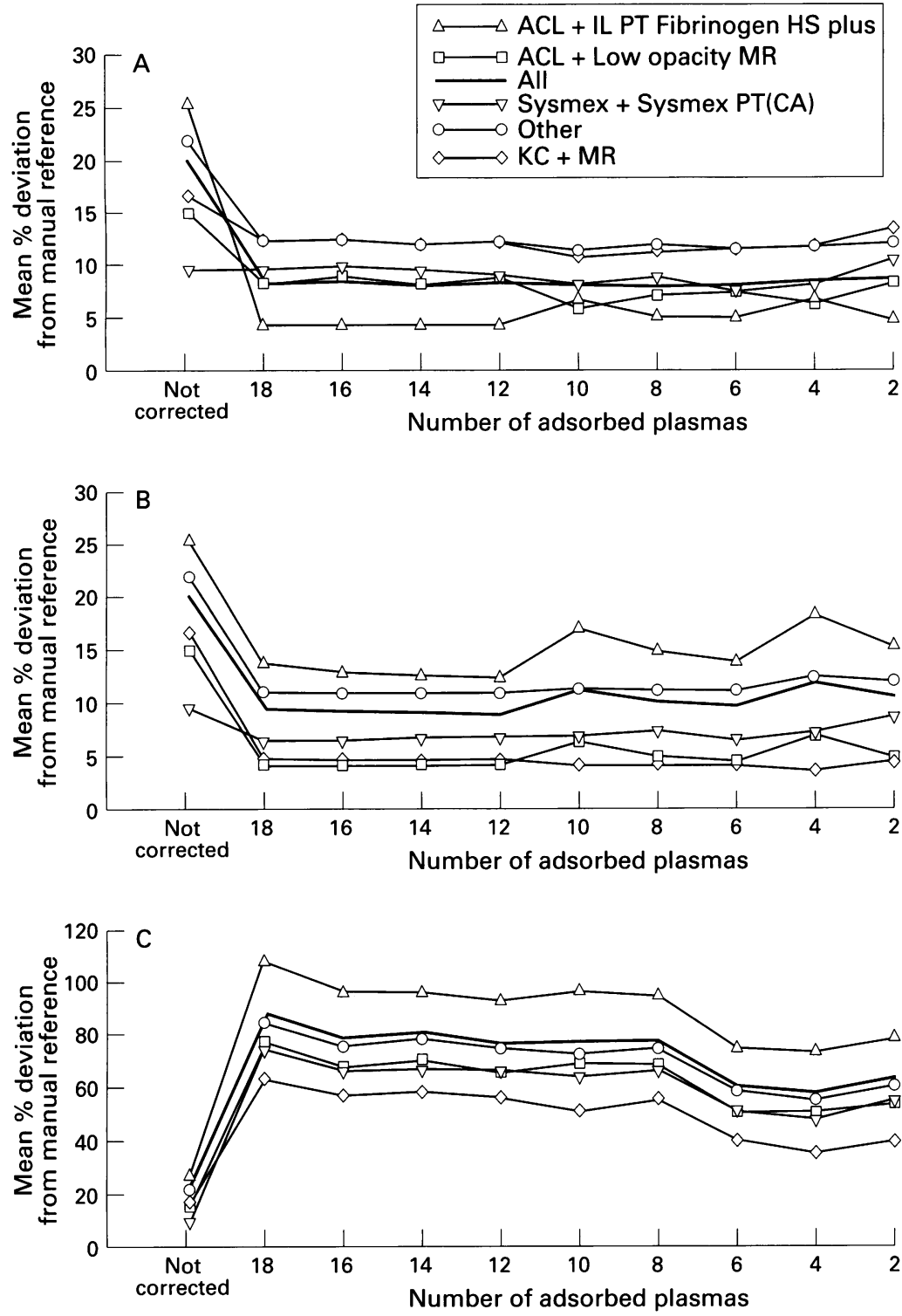

Figure 2 Effect of reducing the number of calibrant plasmas when calibrating the System ISI on the mean percentage deviation of the INR from the manual reference value, using (A) BCT 441, (B) RBT 1303 and (C) RBT 90 as the reference thromboplastins.

thromboplastin calibration procedure. The 20 normal plasmas required by the WHO method are replaced in the calibration by the mean normal prothrombin time (MNPT), which is determined locally by calculating the geometric mean of the prothrombin times of plasmas from at least 20 normal donors.

The present report describes a multicentre study, involving 57 laboratories, to test whether a further reduction in the number of calibrant plasmas is possible without compromising the reliability of the calibration.

\section{Methods}

Fifty seven centres in the UK were asked to determine the prothrombin times, in quadruplicate, of 18 lyophilised adsorbed plasmas and eight frozen coumarin plasmas. The latter were transported to the testing laboratories in dry ice by overnight carrier.

Adsorption onto barium sulphate was used selectively to reduce the vitamin $\mathrm{K}$ dependent clotting factors. Barium sulphate (Sigma, Poole, Dorset, UK) was added to normal plasma, incubated with gentle mixing for 15 minutes and centrifuged at $2000 \times g$ for 10 minutes. Prothrombin time, fibrinogen (by the Clauss technique) and Factor V (by a prothrombin time based assay) were determined, and the entire process repeated until a range of plasmas with prothrombin times spanning the therapeutic range was obtained. If fibrinogen or Factor $\mathrm{V}$ concentrations fell below the normal range the plasma was discarded. Suitable plasmas were dispensed in $0.5 \mathrm{ml}$ aliquots and lyophilised according to a protocol devised at the Thrombosis Reference Centre. The adsorbed plasmas were calibrated manually using IRP BCT 441 (human brain, plain) and RBT 90 (rabbit brain, plain). An in-house rabbit brain preparation, RBT 1303, which had been calibrated previously against BCT 441 and RBT 90, was also used.

Coumarin plasmas were obtained from patients stabilised on oral anticoagulant therapy. These were dispensed in $0.5 \mathrm{ml}$ aliquots and frozen at $-80^{\circ} \mathrm{C}$. These were also calibrated manually using IRP BCT 441 and RBT 90 and the in-house RBT 1303.

Prothrombin times were measured on the local coagulometer/thromboplastin combination and each laboratory provided its system MNPT by calculating the geometric mean of at least 20 normal plasmas.

For each laboratory, the slope of the line relating local system results, including MNPT, to calibrated values obtained with BCT 441, RBT 1303 or RBT 90 was derived by orthogonal regression. The number of adsorbed calibrant plasmas included in the calculation was reduced, two at a time, from 18 to two.

The validity of the System ISI was tested by calculating the percentage deviation from the manual value of the frozen coumarin plasmas (mean of BCT 441 and RBT 90). For each system group, the number of calibrant plasmas used was plotted against the group mean percentage deviation from manual. Group mean coefficients of variation (CV) of System ISI were also calculated.

To determine the influence of calibrant plasma selection on mean \% deviation and $\mathrm{CV}$, four permutations of calibrant plasmas were chosen when the number used in the calibration was 14 or fewer. In each case the selection spanned the therapeutic range.

\section{Results}

Tables 1 and 2 show the system combinations used in the laboratories taking part in the study. The ACL was the most common coagulometer (33/57), followed by Sysmex (13/57). Twenty one centres used Instrumentation Laboratories PT Fibrinogen HS Plus, 16 low opacity Manchester reagent and 11 Manchester reagent. Those combinations with fewer than five users were grouped as others and are described in table 2 .

Figure 1 shows the INR of the plasmas obtained with reference thromboplastins. Panel A reveals the considerable variation in INR of the adsorbed plasmas. BCT 441 gave the tightest range and lowest values (2.05-3.95) 
whereas RBT 90 gave the widest range and highest values (2.2-9.1). Panel B, in contrast, demonstrates the relatively good agreement in


Figure 3 Effect of reducing the number of calibrant plasmas when calibrating the System ISI on the mean CV of the calibration, using (A) BCT 441, (B) RBT 1303 and (C) $R B T 90$ as the reference thromboplastins. See fig 2 for key to symbols.

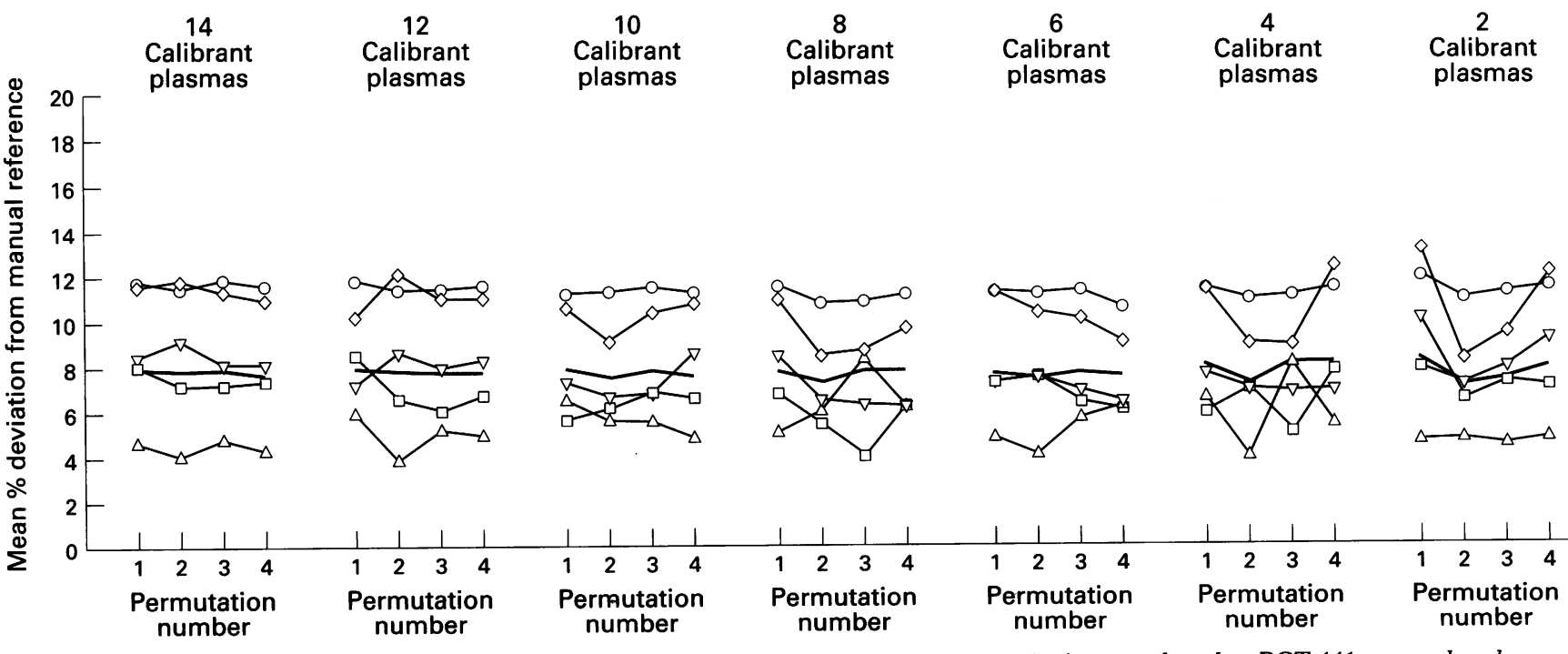

Figure 4 Influence of plasma selection on the mean percentage deviation of the INR from the manual reference value when BCT 441 was used as the reference thromboplastin. See fig 2 for key to symbols.
INR of the frozen coumarin plasmas shown by the thromboplastins used.

All groups of systems tested gave higher INR than those obtained manually with the reference preparations. This mean deviation was $20 \%$ when all the systems were grouped and ranged from $9.34 \%$ with the Sysmex plus Sysmex PT(CA) group to $25.43 \%$ with the ACL plus IL PT Fibrinogen HS Plus group. All system groups, therefore, were in need of correction (figs $2 \mathrm{~A}$ and $2 \mathrm{~B}$ ) and all, apart from the Sysmex plus Sysmex PT(CA) group, benefitted from correction following a System ISI calibration.

The effect on the bias of reducing the number of adsorbed plasmas used to calculate the System ISI is shown in fig 2A with BCT 441 and in fig 2B with RBT 1303 as the manual reference. As the number was reduced from 18 to 12 , there was no change in the percentage deviation. Below 12, inconsistent results were obtained with some systems. With the notable exception of the ACL plus IL PT Fibrinogen HS Plus group, the reduction in bias was more pronounced when RBT 1303 was used. Figure $2 \mathrm{C}$ shows the result of using RBT 90 as the manual reference. In this case the bias increased on System ISI calibration.

Figures $3 \mathrm{~A}, 3 \mathrm{~B}$ and $3 \mathrm{C}$ show the mean $\mathrm{CV}$ of the calibrations using as reference BCT 441, RBT 1303 and RBT 90, respectively. There was no significant trend with reducing numbers of calibrant plasmas for either BCT 441 or RBT 1303, although some inconsistencies appeared at low plasma numbers. There was an increasing trend, starting from a higher $\mathrm{CV}$, shown by RBT 90.

Figure 4 shows the bias obtained with different plasma selections as numbers were reduced, when BCT 441 was used as the reference. Figure 5 gives the results when RBT 1303 was used. When 12 calibrant plasmas were used, plasma selection made little difference. Below 12, inconsistencies appeared in some systems which became more apparent as numbers reduced. Figure 6 shows that when BCT 441 was the reference, the $\mathrm{CV}$ was

$\begin{array}{ccc}6 & 4 & 2 \\ \begin{array}{l}\text { Calibrant } \\ \text { plasmas }\end{array} & \begin{array}{l}\text { Calibrant } \\ \text { plasmas }\end{array} & \begin{array}{l}\text { Calibrant } \\ \text { plasmas }\end{array}\end{array}$




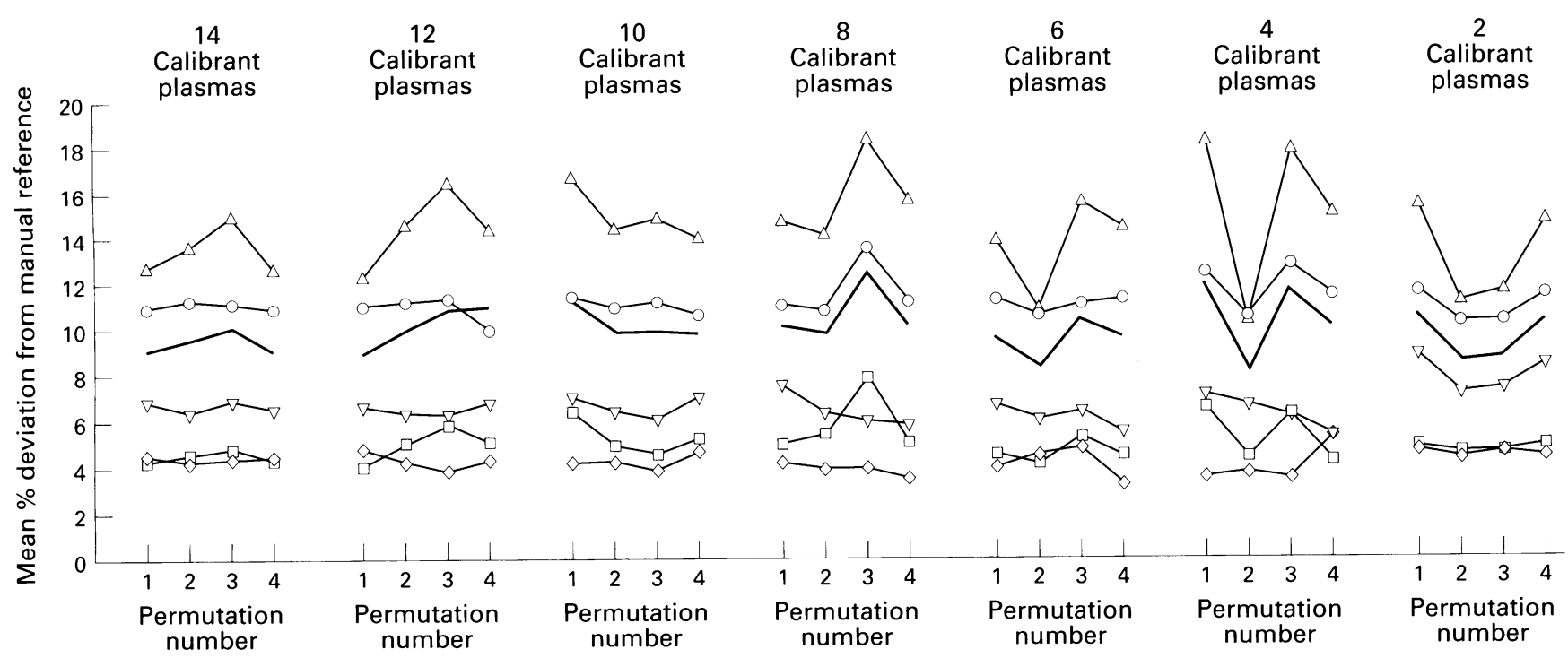

Figure 5 Influence of plasma selection on the mean percentage deviation of the INR from the manual reference value when RBT 1303 was used as the reference thromboplastin. See fig 2 for key to symbols.



Figure 6 Effect of plasma selection in the CV of calibration when BCT 441 was used as the reference thromboplastin. See fig 2 for key to symbols.

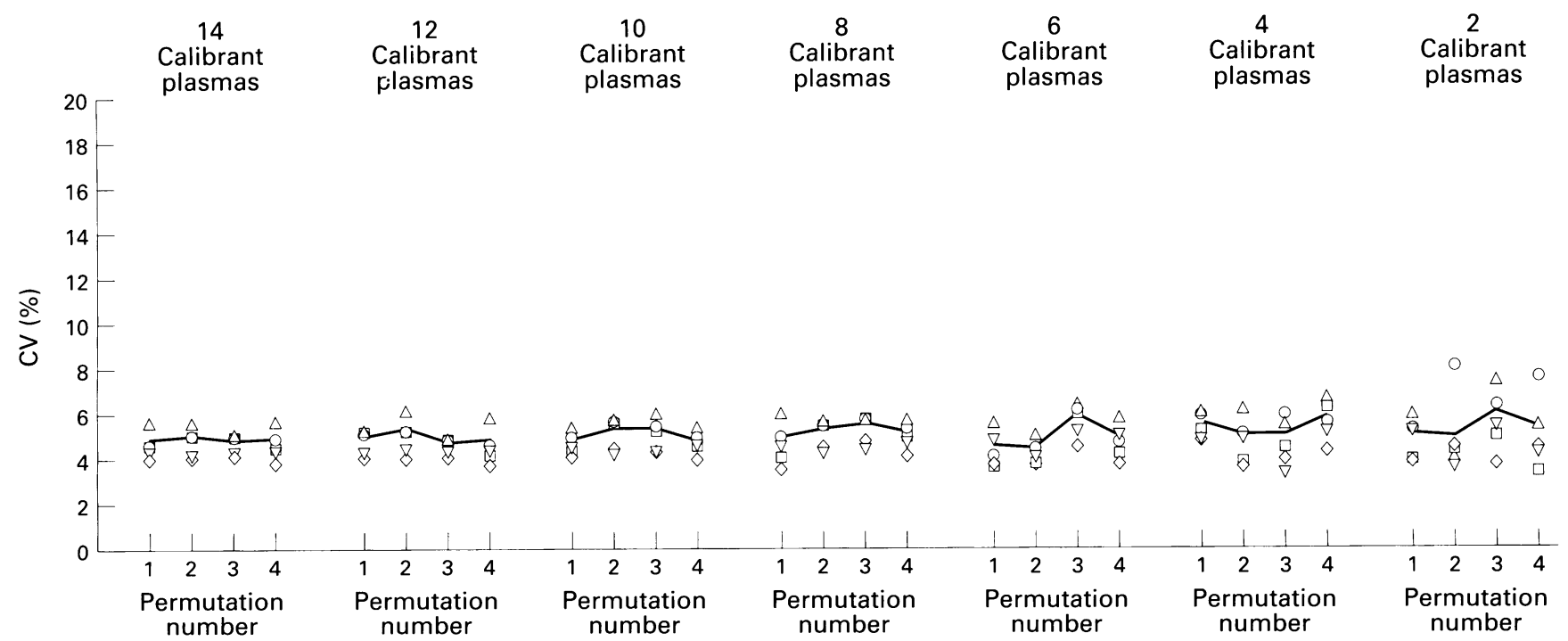

Figure 7 Effect of plasma selection in the CV of calibration when RBT 90 was used as the reference thromboplastin. See fig 2 for key to symbols.

dependent on the plásma selection when six, four and two calibrant plasmas were used. By contrast, when RBT 1303 was the reference (fig 7) there was little change until two calibrant plasmas were used.

\section{Discussion}

According to NEQAS, ${ }^{4}$ the three most widely used coagulometers in the UK are ACL, KC and Sysmex, and the most popular thromboplastin reagents are IL and Manchester. This is 
reflected by the many centres which provided the data for the present study. These were 57 routine hospital laboratories which determined the prothrombin times of the test plasmas according to their standard operating procedures.

As seen in previous studies, all coagulometer/reagent systems showed some deviation in INR from those obtained manually with the reference preparations. ${ }^{56}$ Surprisingly, the ACL and IL combination, which has an instrument specific ISI assigned by the manufacturer, gave the greatest deviation (about $25 \%$ ). In agreement with earlier work ${ }^{78}$ the present study demonstrates the value of assigning a local System ISI in significantly reducing this bias, in this case when BCT 441 or RBT 1303 was used as the manual reference reagent. This was not the case, however, when RBT 90, the current WHO International Reference Preparation for rabbit thromboplastin, was used. It is clear from fig 1 that the interaction of RBT 90 with lyophilised adsorbed plasmas differs greatly from that seen with BCT 441 and RBT 1303. However, as there is relatively good agreement between INR obtained with BCT 441 and RBT 1303, also a rabbit brain reagent, it is unlikely that the lack of sensitivity to protein induced by vitamin $K$ antagonists (PIVKA), reported as a property of rabbit brain thromboplastins, ${ }^{910}$ is wholly responsible for this discrepancy. It may be that RBT 90 is more sensitive to some change in the plasmas following lyophilisation. ${ }^{11}$

The objective of this study was to determine how few calibrant plasmas can be used while still obtaining a reliable System ISI result. Although as few as two calibrant plasmas were sufficient in certain circumstances, it is clear that plasma selection becomes critical when less then 12 are used. The effect of the plasma selection on $\mathrm{CV}$ was less notable when RBT
1303 was used as the reference. These observations are based on the use of individual plasmas from single sources. It is possible, however, that plasma pools from multiple donors may reduce the biological variation and permit the use of fewer calibrants. As the majority of the participants used rabbit brain reagents, this gives further support to the hypothesis that calibrations should be of like with like species.

In conclusion, the use of fewer than 12 calibrant plasmas for System ISI calibrations cannot be recommended.

1 Clarke K, Taberner DA, Thomson JM, Morris JA, Poller L. Assessment of value of calibrated lyophilised plasmas to determine International Sensitivity Index for coagulometers. 7 Clin Pathol 1992;45:58-60.

2 Poller L, Thomson JM, Taberner DA, Clarke DK. The correction of coagulometer effects on international normalised ratios: a multicentre evaluation. Br f Haematol 1994;86: 112-17.

3 WHO Expert Committee on Biological Standardisation. 33rd Report. World Health Organ Tech Rep Ser 1983;687: 81-105.

4 UK NEQAS for Blood Coagulation. Report to participants on surveys 92 and 93. Shefield: NEQAS, December 1995.

5 D'Angelo A, Seveso MP, D'Angelo SV, Gilardoni F, Macagni A, Manotti C, et al. Comparison of two automated coagulometers and the manual tilt tube method for the determination of prothrombin time. $\mathrm{Am} \mathrm{f}$ Clin Pathol 1989;92:321-8.

6 Poggio M, van den Besselaar AMHP, van der Velde EA, Bertina RM. The effect of some instruments for prothrombin time testing on the International Sensitivity Index (ISI) of two rabbit tissue thromboplastin reagents. Thromb Haemost 1989;62:868-74.

7 Chantarangkul V, Tripodi A, Mannucci PM. The effect of instrumentation on thromboplastin calibration. Thromb Haemost 1992;67:588-9.

8 Ray MJ, Smith IR. The dependence of the International Sensitivity Index on the coagulometer used to perform the prothrombin time. Thromb Haemost 1990;63:424-9.

9 Denson KWE. Thromboplastin - sensitivity, precision and other characteristics. Clin Lab Haematol 1988;10:315-28.

10 Hemker HC, Veltkamp JJ, Loeliger EA. Kinetic aspects of the interaction of blood clotting enzymes. III. Demonstration of the existence of an inhibitor of prothrombin convertion of the existence of an inhibitor of prothrombin conver-
sion in vitamin $\mathrm{K}$ deficiency. Thrombosis et Diathesis sion in vitamin K deficiency.

11 Craig S, Dufty JMK, Stevenson KJ, Goodman L, Leeming DR, Taberner DA. The effect of freezing and freeze-drying coumarinised plasma on International Normalised Ratio. Blood Coag Fibrinol 1995;6:177. 